\title{
Performance of a beam-multiplexing diamond crystal monochromator at the Linac Coherent Light Source
}

Zhu, Diling; Feng, Yiping; Stoupin, Stanislav; Terentyev, Sergey A.; Lemke, Henrik T.; Fritz, David M.; Chollet, Matthieu; Glownia, J.M.; Alonso-Mori, Roberto; Sikorski, Marcin

Total number of authors:

18

Published in:

Review of Scientific Instruments

Link to article, DOI:

$10.1063 / 1.4880724$

Publication date:

2014

Document Version

Publisher's PDF, also known as Version of record

Link back to DTU Orbit

Citation (APA):

Zhu, D., Feng, Y., Stoupin, S., Terentyev, S. A., Lemke, H. T., Fritz, D. M., Chollet, M., Glownia, J. M., AlonsoMori, R., Sikorski, M., Song, S., Brandt van Driel, T., Williams, G. J., Messerschmidt, M., Boutet, S., Blank, V. D., Shvyd'ko, Y. V., \& Robert, A. (2014). Performance of a beam-multiplexing diamond crystal monochromator at the Linac Coherent Light Source. Review of Scientific Instruments, 85(6), 063106.

https://doi.org/10.1063/1.4880724

\section{General rights}

Copyright and moral rights for the publications made accessible in the public portal are retained by the authors and/or other copyright owners and it is a condition of accessing publications that users recognise and abide by the legal requirements associated with these rights.

- Users may download and print one copy of any publication from the public portal for the purpose of private study or research.

- You may not further distribute the material or use it for any profit-making activity or commercial gain

- You may freely distribute the URL identifying the publication in the public portal 


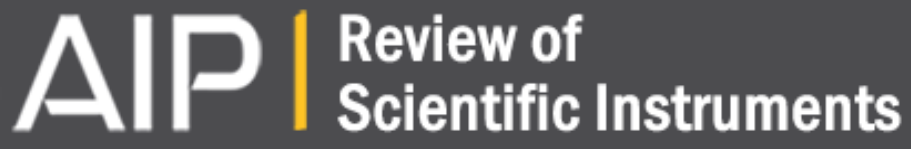

\section{Performance of a beam-multiplexing diamond crystal monochromator at the Linac Coherent Light Source}

Diling Zhu, Yiping Feng, Stanislav Stoupin, Sergey A. Terentyev, Henrik T. Lemke, David M. Fritz, Matthieu Chollet, J. M. Glownia, Roberto Alonso-Mori, Marcin Sikorski, Sanghoon Song, Tim B. van Driel, Garth J. Williams, Marc Messerschmidt, Sébastien Boutet, Vladimir D. Blank, Yuri V. Shvyd'ko, and Aymeric Robert

Citation: Review of Scientific Instruments 85, 063106 (2014); doi: 10.1063/1.4880724

View online: http://dx.doi.org/10.1063/1.4880724

View Table of Contents: http://scitation.aip.org/content/aip/journal/rsi/85/6?ver=pdfcov

Published by the AIP Publishing

\section{Articles you may be interested in}

The soft x-ray instrument for materials studies at the linac coherent light source x-ray free-electron laser Rev. Sci. Instrum. 83, 043107 (2012); 10.1063/1.3698294

X-ray-optical cross-correlator for gas-phase experiments at the Linac Coherent Light Source free-electron laser Appl. Phys. Lett. 100, 121107 (2012); 10.1063/1.3695163

Linac Coherent Light Source soft $\mathrm{x}$-ray materials science instrument optical design and monochromator commissioning

Rev. Sci. Instrum. 82, 093104 (2011); 10.1063/1.3633947

Xray Optics Research for Linac Coherent Light Source: Interaction of Ultrashort Xray Pulses with Matter AIP Conf. Proc. 641, 596 (2002); 10.1063/1.1521083

The coherent light source project at SLAC

AIP Conf. Proc. 525, 623 (2000); 10.1063/1.1291979

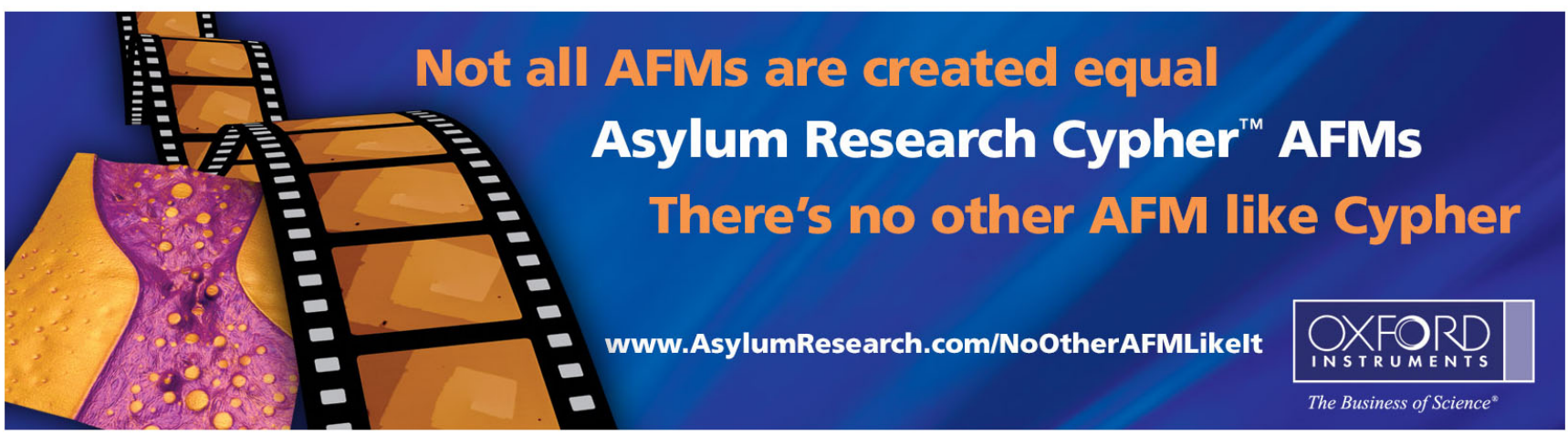




\title{
Performance of a beam-multiplexing diamond crystal monochromator at the Linac Coherent Light Source
}

\author{
Diling Zhu, ${ }^{1, a)}$ Yiping Feng, ${ }^{1}$ Stanislav Stoupin, ${ }^{2}$ Sergey A. Terentyev,${ }^{3}$ Henrik T. Lemke, ${ }^{1}$ \\ David M. Fritz, ${ }^{1}$ Matthieu Chollet, ${ }^{1}$ J. M. Glownia, ${ }^{1}$ Roberto Alonso-Mori, ${ }^{1}$ Marcin Sikorski, ${ }^{1}$ \\ Sanghoon Song, ${ }^{1}$ Tim B. van Driel, ${ }^{1,4}$ Garth J. Williams, ${ }^{1}$ Marc Messerschmidt, ${ }^{1}$ \\ Sébastien Boutet, ${ }^{1}$ Vladimir D. Blank, ${ }^{3}$ Yuri V. Shvyd'ko, ${ }^{2}$ and Aymeric Robert ${ }^{1}$ \\ ${ }^{1}$ Linac Coherent Light Source, SLAC National Accelerator Laboratory, Menlo Park, California 94025, USA \\ ${ }^{2}$ Advanced Photon Source, Argonne National Laboratory, Lemont, Illinois 60439, USA \\ ${ }^{3}$ Technological Institute of Superhard and Novel Carbon Materials, Tsentralnaya str. 7a, Troitsk, \\ Moscow 142190, Russia \\ ${ }^{4}$ Center for Molecular Movies, Department of Physics, Technical University of Denmark, \\ DK-2800 Lyngby, Denmark
}

(Received 5 December 2013; accepted 19 May 2014; published online 6 June 2014)

\begin{abstract}
A double-crystal diamond monochromator was recently implemented at the Linac Coherent Light Source. It enables splitting pulses generated by the free electron laser in the hard x-ray regime and thus allows the simultaneous operations of two instruments. Both monochromator crystals are HighPressure High-Temperature grown type-IIa diamond crystal plates with the (111) orientation. The first crystal has a thickness of $\sim 100 \mu \mathrm{m}$ to allow high reflectivity within the Bragg bandwidth and good transmission for the other wavelengths for downstream use. The second crystal is about $300 \mu \mathrm{m}$ thick and makes the exit beam of the monochromator parallel to the incoming beam with an offset of $600 \mathrm{~mm}$. Here we present details on the monochromator design and its performance. (C) 2014 AIP Publishing LLC. [http://dx.doi.org/10.1063/1.4880724]
\end{abstract}

\section{INTRODUCTION}

The successful operation of x-ray Free Electron Lasers (FEL) in the hard $\mathrm{x}$-ray regime opened up the possibility of a broad spectrum of new experiments. ${ }^{1,2}$ Numerous applications in atomic physics, astrophysics, condensed matter physics, material science, chemistry, and biological sciences have already generated a plethora of interesting observations and novel measurement techniques that will have long-lasting impact to the scientific community. ${ }^{3-14}$ The growing enthusiasm in the application of $\mathrm{x}$-ray FELs is reflected in the continuous increase in the number of experiment proposals requesting beamtime. Currently, with a single undulator in operation, only a single instrument at a time can perform an experiment. This limits the total number of experiments and raises the operation cost per experiment considerably as compared with synchrotron sources where several tens of beamlines, each with its own independent insertion device, operate simultaneously.

The concept of splitting x-ray beams with thin diamond crystals to allow simultaneous experiments has been previously implemented at the Troika beamline at the European Synchrotron Radiation Facility ${ }^{15}$ and the Hyogo beamline at SPring-8. ${ }^{16}$ A similar scheme was incorporated in the instrument design and layout of the Linac Coherent Light Source (LCLS) from the very beginning, as shown in Fig. 1. Two Large Offset Double-Crystal Monochromators (LODCMs) were engineered, manufactured, and installed (JJ X-Ray, Denmark) along the hard X-ray beamline: one in the X-ray Pump Probe (XPP) instrument hutch in the Near Experiment Hall

\footnotetext{
a) Author to whom correspondence should be addressed. Electronic mail dlzhu@slac.stanford.edu
}

(NEH) and the other in the X-Ray Tunnel (XRT) between the NEH and the Far Experimental Hall (FEH). Both LODCMs are designed to provide a monochromatic beam to an instrument with a $600 \mathrm{~mm}$ offset from the main beam. The beamline components of the XPP instrument and the X-ray Correlation Spectroscopy (XCS) instrument can all be translated for operation in their offset positions. ${ }^{17} \mathrm{Si}(111)$ crystal pairs were installed and commissioned for both LODCMs since 2011 and are regularly used in experiments that required a narrower bandwidth for energy and $q$ resolution, or longitudinal coherence considerations. ${ }^{18}$ This also provides flexibility in experiment scheduling as the switching between experiments in different hutches can be simply done by inserting the crystal at the press of a button rather than shifting the whole instrument in and out of the pink beam path, which typically takes several hours. With a high quality thin transmissive crystal as the first LODCM crystal as shown in Fig. 2, two branch lines, one pink and the other monochromatic, can be used to allow simultaneous experiments in different hutches and thereby increase available beamtime. The LODCM in XPP can then allow simultaneous experiments in XPP with any of the three instruments located downstream in the FEH: (i) to XCS by using the LODCM located in the XRT; (ii) to the Coherent Xray Imaging (CXI) instrument; ${ }^{19}$ (iii) to the Matter in Extreme Conditions (MEC) instrument by inserting the MEC mirror in the beampath. The second LODCM in the XRT splits the beam between XCS and CXI, potentially enabling three simultaneous experiments.

Silicon was initially considered as a candidate for the beam-splitting crystal as it was easily accessible in different orientations. Mature fabrication processes exist to thin and polish them with minimal impact to the crystalline quality. 


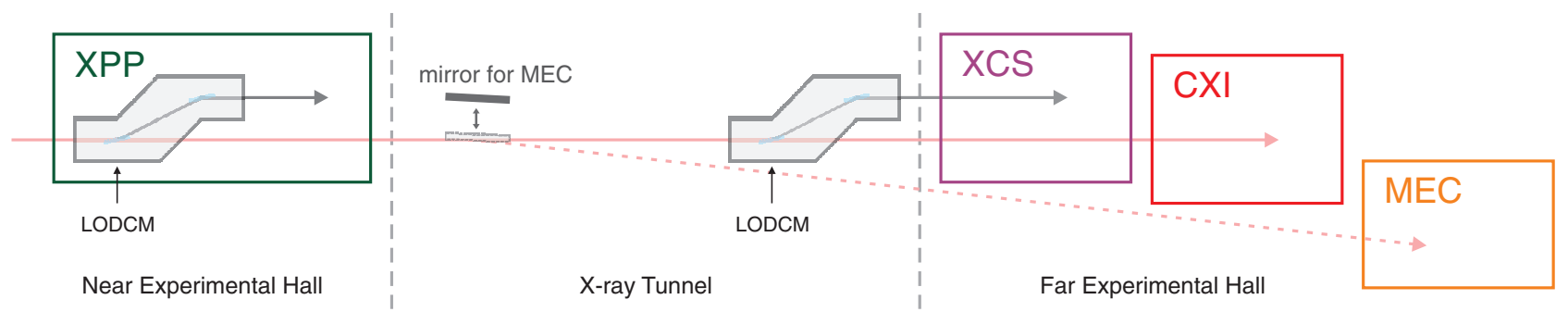

FIG. 1. LCLS hard x-ray instrument layout. The XPP LODCM is located in the NEH. The XCS instrument LODCM is located in the XRT. The three instruments in the FEH are XCS, CXI, and MEC.

However, due to its higher $Z$, thus larger absorption cross section at the typical LCLS hard x-ray photon energy range of 4-10 keV, a crystal thickness on the order of 5-10 $\mu \mathrm{m}$ is needed to provide reasonable transmission $(>50 \%)$. This leads to mechanical rigidity issues arising from the impulsive energy deposition of the $120 \mathrm{~Hz}$ FEL pulses and the sequential thermal expansion and relaxation. Severe distortions were observed in the diffracted beam profile when the crystal was illuminated with FEL pulses at milliJoule $(\mathrm{mJ})$ levels. ${ }^{20}$ Diamond $\left(\mathrm{C}^{*}\right)$ on the other hand was always an attractive option thanks to its lower absorption cross section, superior thermal conductivity, and lower thermal expansion coefficient. The challenge as previously discovered at 3rd generation synchrotron sources is in the crystal growth, the thin crystal plate fabrication, and mounting. ${ }^{21-23}$ In order to maximize the photon flux in the monochromatic branch, the (111) orientation is preferred, which happens to be a more difficult orientation to fabricate and polish than (100) and (110) with low miscut. Recent improvements in High Pressure High-Temperature (HPHT) synthesis processes have shown promising results in improved crystal quality. ${ }^{24-29}$ A set of diamond crystals of the (111) orientation, mounted on an all-diamond mounting frame assembly, was prepared by the Technological Institute for Superhard and Novel Carbon Materials (TISNCM, Troisk, Russia). They were characterized and optimized at the Advanced Photon Source (APS, Argonne, IL) by high resolution topography and then installed in the XPP LODCM for splitting the FEL beam. In this paper, we report the performance of this beam-multiplexing thindiamond monochromator.

\section{MONOCHROMATOR DESIGN}

The LODCMs use a symmetric double Bragg reflection geometry similar to the LOM1250 installed at the P08

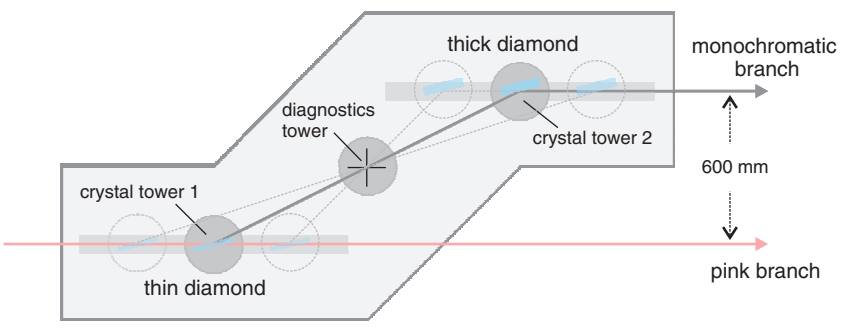

FIG. 2. Top view of the symmetric diffraction geometry of the LODCM. The red solid line indicates the main LCLS (pink) branch. The black solid line indicates a monochromatic branch configuration delivering the beam to XPP. The dashed lines show two other potential monochromatic configurations delivering higher and lower photon energies to XPP. instrument $^{30}$ at PETRA III. However, instead of vertically offsetting the beam, a horizontal diffraction geometry was chosen to allow the beamline components in XPP and XCS, mounted on horizontal translation rails, to be easily translated into either the pink or the monochromatic branch. As shown in Fig. 2, the fixed $600 \mathrm{~mm}$ beam offset is achieved by two Bragg reflections.

In this symmetric design, the diffracted beam from the first crystal always passes through the center of symmetry of the LODCM chamber, where beam diagnostics are installed for alignment and calibration purposes. The crystal towers 1 and 2 both provide the necessary degrees of freedom in translation and rotation for positioning and orienting each crystal. Additional motions allow different crystals or different parts of the same crystal to be translated into the operating position. The diagnostic tower at the center of symmetry of the chamber incorporates features to ensure correct alignment and calibration. A set of horizontal and vertical slits can be inserted to ensure the first reflection travels through the center of symmetry and also at the correct beam height. There is also a scintillator screen for beam profile inspection. A set of energy calibration filters ( $\mathrm{Mn}, \mathrm{Fe}, \mathrm{Ni}, \mathrm{Cu}, \mathrm{Zr}$, etc.) are mounted on a filter wheel. A motorized diode behind the filter wheel can be used for spectrum measurements in either transmission or fluorescence mode to calibrate the absolute photon energy.

Currently, two pairs of crystals are installed and commissioned in the XPP LODCM: a pair of thick Si(111) crystals and a pair of thin $C^{*}(111)$ crystals. The arrangement is shown in Fig. 3 for the first crystal tower. Both Si crystals have

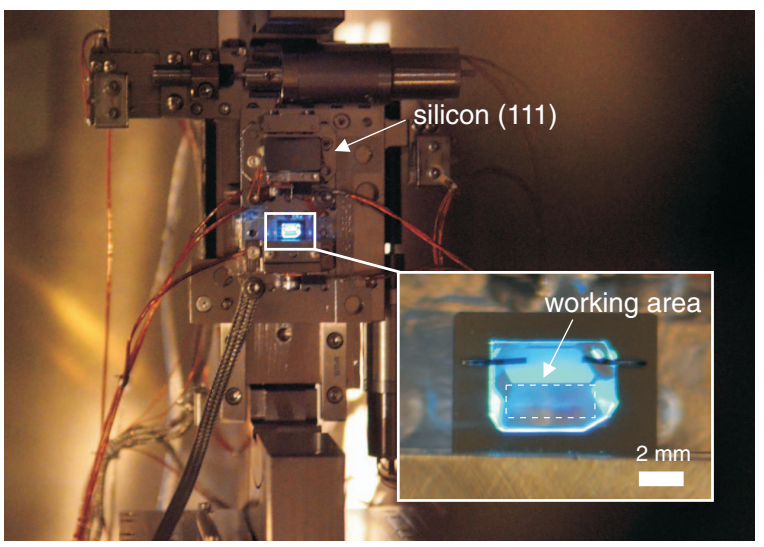

FIG. 3. Photo of the first crystal tower in operation with the thin diamond crystal in the FEL beam. The silicon crystal sits above as indicated. Inset is a magnified view of the diamond crystal while illuminated by $\mathrm{x}$-rays, with the working area indicated by the dashed area. 
dimensions of $20 \times 12 \times 9 \mathrm{~mm}^{3}$. Both diamond crystals have lateral dimensions of about $5 \times 5 \mathrm{~mm}^{2}$ with a $2 \times 4 \mathrm{~mm}^{2}$ working area. The blue color of the diamond as seen in Fig. 3 originates from the x-ray induced luminescence of impurities in the diamond crystal, from which growth sectors with different impurity concentrations can be identified. ${ }^{24}$ The diamond luminescence can be used to identify the location of the beam on the crystal and help the initial alignment. The first diamond crystal has a thickness of $\sim 100 \mu \mathrm{m}$ and the second of $\sim 300 \mu \mathrm{m}$. The accessible diffraction angle is determined by the length of the two linear translation rails at the base of the crystal towers. An angular range of $14^{\circ}-90^{\circ}$ can be reached, corresponding to energy ranges of $3-16 \mathrm{keV}$ and $4-25 \mathrm{keV}$ for $\mathrm{Si}(111)$ and $\mathrm{C}^{*}(111)$, respectively. Due to the horizontal linear polarization of the LCLS beam, the diffraction efficiency drops significantly when approaching $90^{\circ}$ diffraction angle. The typical hard x-ray energy range of the LCLS fundamental wavelengths that are used in experiments $(5-11 \mathrm{keV})$ is well covered by both crystal sets.

Even if LCLS delivers pulses with energy up to a few $\mathrm{mJ}$ at $120 \mathrm{~Hz}$ repetition rate, the beam presents a relatively moderate average thermal load on the first crystal $(<1 \mathrm{~W})$. Therefore, no active cooling was implemented for any crystals. However, fluctuations in FEL output pose a big challenge for the stability. Beam pointing drifts in the monochromatic branch on the order of 1-2 $\mu$ rad were observed when using both $\mathrm{Si}$ or $\mathrm{C}^{*}$ crystals. The observed drift is strongly correlated with the FEL output level, which is by far less stable comparing to the synchrotron sources. For example, the beam can go down for a few seconds or minutes due to a linac issue, and the brief outage will then cause a beam pointing shift of $1-2 \mu \mathrm{rad}$ after the beam is recovered. The pointing could then take a few minutes to reach the previous location as thermal equilibrium is reestablished. The pointing is much more stable when the FEL output remains at a steady level. To date, the level of drift has been acceptable for most user experiments requesting monochromatic beam. When higher degree of beam stability is required, the experiment can be scheduled to run in the pink branch with a standard channel cut monochromator.

The first thin crystal is central to the multiplexing operation of the LODCM. Its single-crystalline quality directly couples to the level of performance of the diffracted and transmitted beams. Two aspects are particularly challenging: (i) to thin down and polish a single crystal diamond plate to $100 \mu \mathrm{m}$ thickness without introducing defects; (ii) to mount the crystal with sufficient thermal contact to unload the absorbed $\mathrm{x}$-ray energy without introducing additional strain. The crystals were grown at the TISNCM using HPHT synthesis method starting from a (001) seed. They were then laser cut into thin plates. Polishing was performed with an intentional $2^{\circ}$ miscut for optimal surface quality. The working area originated for the most part from the (001) growth sector with a low concentration of defects such as minor dislocations and micro-inclusions. We initially used a graphite strain free mount previously developed for the LCLS selfseeding monochromator. ${ }^{29,31}$ Due to poor thermal contacts of this mount, a crystal temperature increase up to $100{ }^{\circ} \mathrm{C}$ was observed when illuminated with $1 \mathrm{~mJ}, 8 \mathrm{keV}$ LCLS pulses
$400 \mu \mathrm{m}$ FWHM in size at full beam rate, using a thermal imaging camera. ${ }^{32}$

A new mount designed and fabricated by the TISNCM was used to increase the thermal contact while minimizing the mounting strain. The assembly consists a $500 \mu \mathrm{m}$ thick CVD (chemical vapor deposition) diamond substrate frame (black rectangular frame behind the diamond crystal shown in the inset of Fig. 3), and two perforated CVD diamond micro-clips with low spring constant for pressing the thin crystal onto the frame. The CVD diamond frames were clamped to the stainless steel monochromator crystal mounts. The all diamond design significantly improved the thermal conduction between the thin crystal and the mounting frame. Under typical FEL beam condition the observed temperature increase was well below $10{ }^{\circ} \mathrm{C}$. The mounted thin diamond plates were characterized at the x-ray laboratory at APS using double crystal topography in rocking curve imaging mode. It was confirmed that the working area of the crystal is nearly defect free, with only a few microinclusions. The mounting strain was minimized to about $1.5 \mu \mathrm{rad}$ over the working area by adjusting the micro-clips. More detail of the mounting and crystal characterization is beyond the scope of the current paper and is discussed extensively in Ref. 33. Section III of the paper will focus on the performance of the LODCM with $\mathrm{C}^{*}(111)$ crystals.

\section{PERFORMANCE}

The performances of a Bragg beam-splitting thin crystal depends on three requirements: (i) the incoming bandwidth is larger than the crystal reflection bandwidth; (ii) the thickness of the crystal is comparable to or larger than the extinction depth of the Bragg reflection; and (iii) the thickness of the crystal is much smaller than the penetration depth of the other wavelengths. LCLS currently operates in the SASE (self amplified spontaneous emission) mode for most experiments. ${ }^{1}$ The shot-averaged bandwidth of the FEL output in the hard xray regime is typically $\Delta E / E=0.4 \% \mathrm{FWHM}$, due to the natural bandwidth from the SASE process $(0.3 \%)$ and the shot-toshot electron beam energy jitter. This is much broader than the $\sim 0.005 \%$ bandwidth of the $\mathrm{C}^{*}$ (111) reflection. The thickness of $100 \mu \mathrm{m}$ is much larger than the extinction depth over the operation photon energy range, leading to a reflectivity within the diffraction bandwidth that is close to unity. Assuming that the average spectral distribution of the FEL is Gaussian with a width of $30 \mathrm{eV}$ centered at $8.0 \mathrm{keV}$, a $0.4 \mathrm{eV}$ reflection bandwidth placed at the center of the FEL spectrum will reflect on average $1.4 \%$ of the total incident pulse energy. This value typically varies between $1 \%-2 \%$ depending on the machine operation conditions. The other wavelengths will propagate through the thin diamond with some absorption losses.

The spectrum of the pink branch is measured using a bent crystal single-shot spectrometer at the XPP instrument. ${ }^{34}$ As illustrated in Fig. 4(a), the spectrum of a typical single-shot consists of many random spikes due to the stochastic nature of the SASE process. It is only in the shot-averaged spectrum that the spectral notch created by the diamond reflection becomes clear. The spectral notch width is determined by the bandwidth of the $C^{*}(111)$ reflection. It was measured to be 
(a)
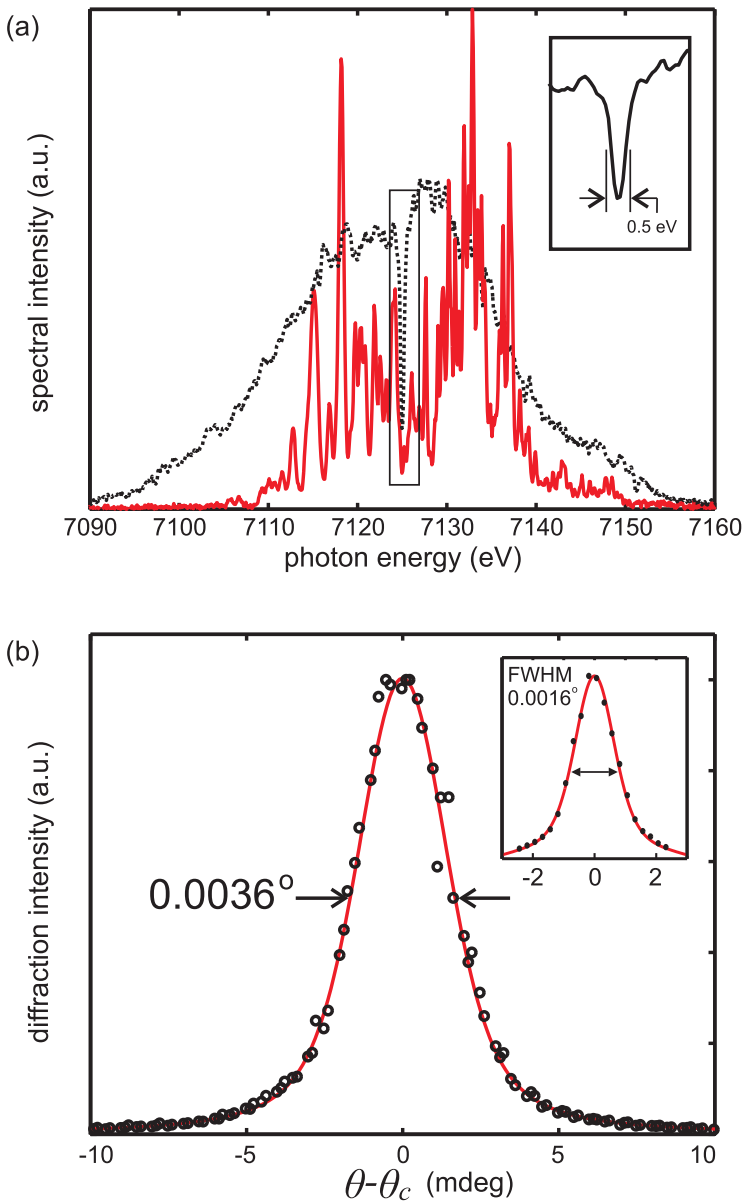

FIG. 4. (a) Single-shot (solid line, red) and 240-shot average (dotted) spectrum of the pink branch after the thin diamond. The inset is a zoomed view of the spectral notch seen in the averaged spectrum. (b) Monochromatic branch bandwidth characterized by rocking curve measurement using a $\mathrm{Si}(440)$ analyzer at $7.9 \mathrm{keV}$. Inset shows the rocking curve of the second diamond crystal. For both plots, measurements are shown as symbols and a Voigt functions are used to model the data (line)

$\sim 0.5 \mathrm{eV}$ as indicated in the inset of Fig. 4(a). The width of this notch is a first indication of good crystal quality. The small amount of broadening and the incomplete extinction is due to the limited resolution of the spectrometer.

The reflected beam goes through another reflection from the second (thicker) diamond crystal, and is sent downstream into the XPP instrument in its $600 \mathrm{~mm}$ offset position. We measured the bandwidth of the monochromatic branch using a $\mathrm{Si}(440)$ crystal as an analyzer downstream in the vertical reflection geometry. A rocking curve was measured at $7.9 \mathrm{keV}$ and is shown in Fig. 4(b). The $\mathrm{Si}(440)$ bandwidth is much narrower than that of the $C^{*}(111)$. Therefore, the measured width is dominated by the bandwidth of the diamond monochromator upstream. The measured $3.6 \mathrm{mdeg}$ (FWHM) correspond to $0.34 \mathrm{eV}$ at $7.9 \mathrm{keV}$, in agreement with the calculated value of $0.33 \mathrm{eV}$, where both the diffraction geometry and a $2 \mu \mathrm{rad}$ beam divergence of the FEL were taken into account. In addition, the inset in Fig. 4 shows that the rocking curve of the second diamond crystal at $7.9 \mathrm{keV}$ has also a width closely matching the calculated value of $1.4 \mathrm{mdeg}$. The minimal broadening observed in the rocking curve provides direct evidence of the high quality of both diamond crystals as well as the absence of substantial crystal strain.

Another important indicator of the performance of the beam-sharing monochromator is the throughput in both the pink and the monochromatic branches. In Fig. 5(a) we compare the throughput of the pink branch, quantified by the measured transmission of the thin diamond crystal, with calculation. The uncertainty band of the calculation, shown as the gray band in Fig. 5(a), takes into consideration the error bar from the crystal thickness measurement and the crystal mis-cut. The throughput of the monochromatic branch is harder to quantify as the number of photons that gets reflected depends strongly on many details of the FEL setup (e.g., electron beam compression level, electron energy jitter). We thus compare it to what is obtained with a thick silicon crystal. Fig. 5(b) shows the throughput of the monochromatic branch as compared to the thick $\mathrm{Si}(111)$ crystal configuration at the same photon energy. The throughput is lower for diamond and it is mainly due to its smaller Darwin width. The theoretical throughput ratio is calculated considering both bandwidths and reflectivities and is plotted with the solid line. Each experimental data point was measured within a few hours when the FEL was operating in very similar conditions (using the inten-
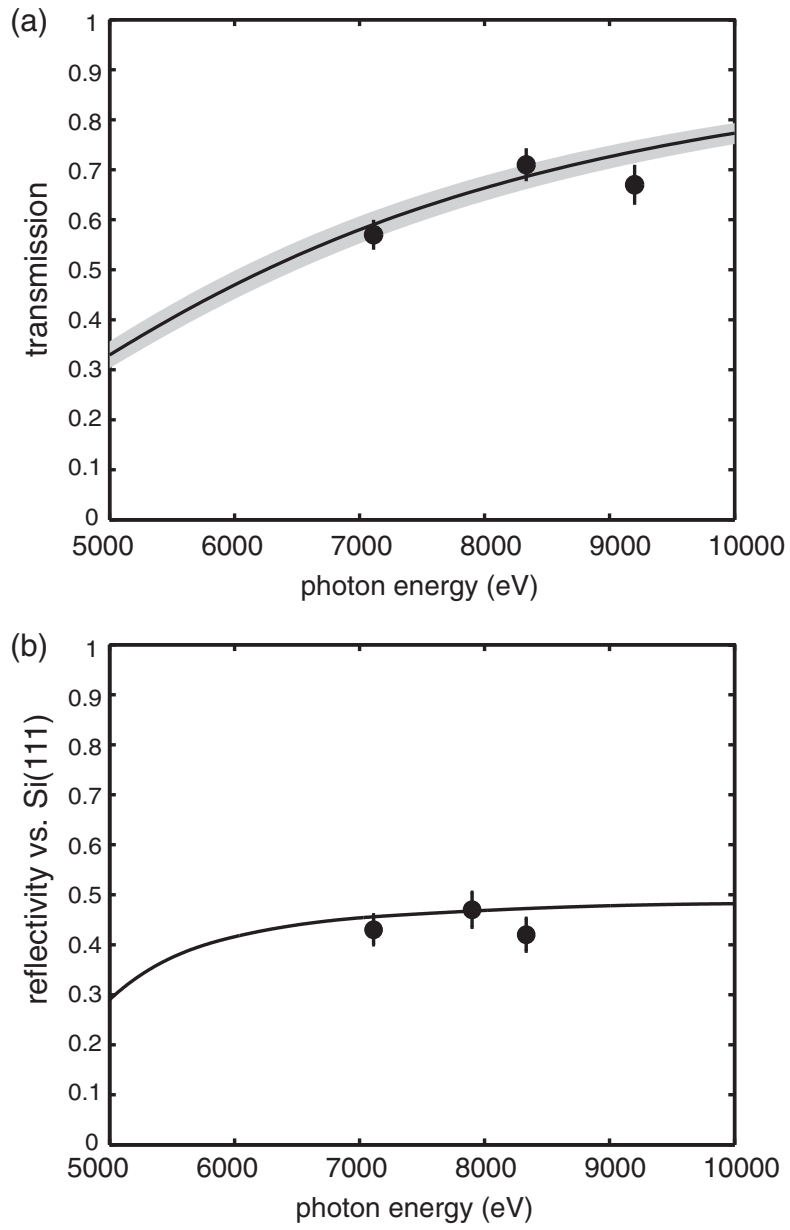

FIG. 5. Theoretical (solid line) and measured (symbol) monochromator throughput for both the pink and the monochromatic branches. (a) Transmission of the thin $\mathrm{C}^{*}$ crystal. The gray band indicates the calculation uncertainty. (b) Monochromatic branch throughput, normalized to $\mathrm{Si}(111)$ for both the calculation and the measurement. 


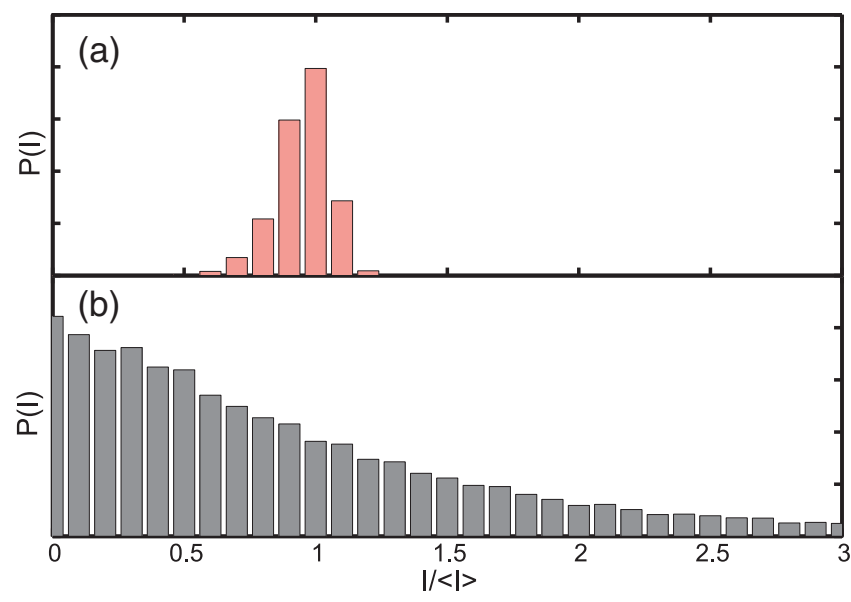

FIG. 6. Normalized shot-to-shot intensity distribution for the pink (a) and monochromatic (b) branches.

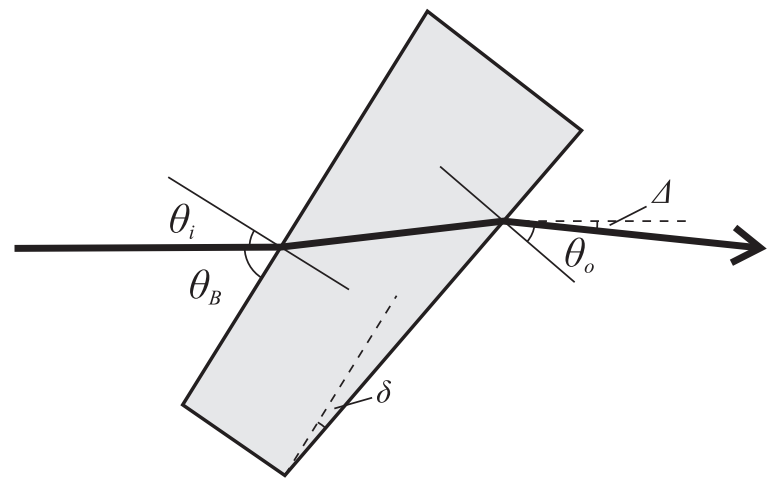

FIG. 7. Refraction geometry of the beam steering by the diamond crystal. $\theta_{B}$ is the Bragg angle, $\theta_{i}$ and $\theta_{o}$ are the incidence and exit angle of the beam from the surface normal. $\delta$ is the wedge angle, and $\Delta$ is the steering angle.
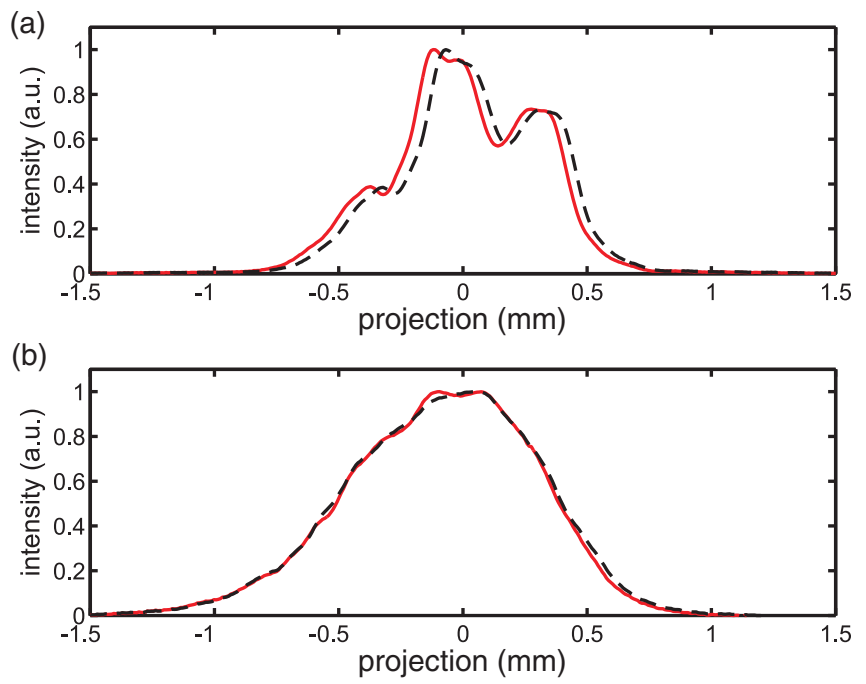

FIG. 8. Horizontal (a) and vertical (b) projections of the beam profile measured 200 meter downstream of the XPP LODCM in the pink branch, with (red solid) and without (black dashed) the diamond crystal. sity monitors at XPP normalized by the gas monitor upstream of the LODCM which tracks the incoming pulse energy ${ }^{35}$ ). Both branches perform in agreement with calculation and therefore demonstrate again the high single crystal quality of the diamond crystal pair.

As an example, at $8 \mathrm{keV}$, when the FEL operates at $1 \mathrm{~mJ}$ (i.e., equivalent to $\sim 10^{12}$ photons per pulse ( $\mathrm{ph} / \mathrm{pulse}$ )), the pink branch receives $\sim 6 \times 10^{11} \mathrm{ph} / \mathrm{pulse}$, and the monochromatic branch on the order of $10^{10} \mathrm{ph} /$ pulse on average. The shot-to-shot pulse energy distribution of the monochromatic branch differs significantly from that of the pink branch. As shown in Fig. 6, while the pink branch still maintains a small variance in pulse energy around its average value, the monochromatic branch shows nearly $100 \%$ fluctuation as one would expect from the SASE spectral fluctuation. ${ }^{18}$

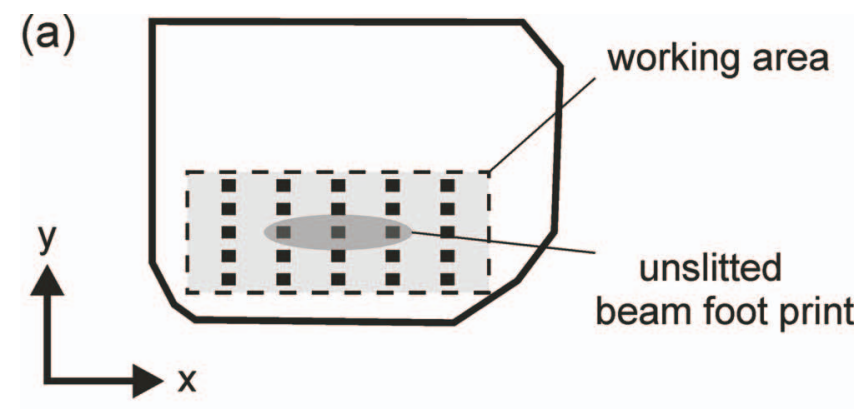

(b)

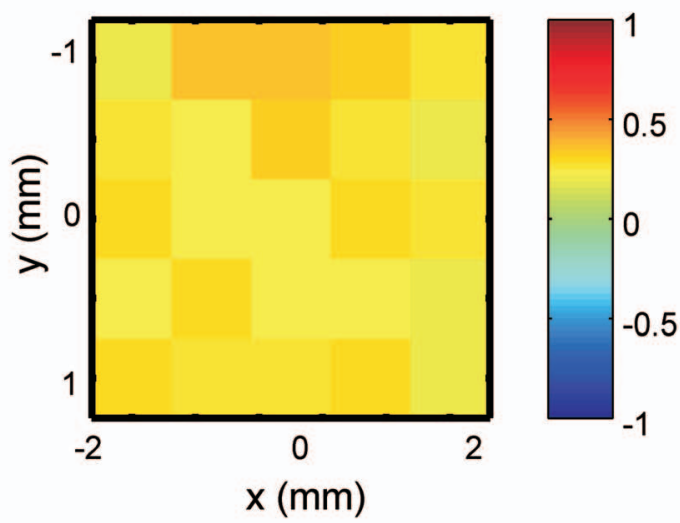

(c)

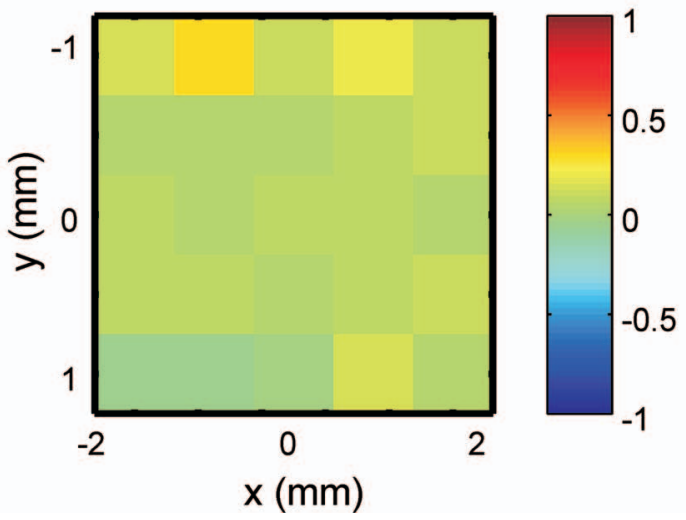

FIG. 9. (a) Locations on the diamond where beam steering were measured. (b) and (c) display the 2D maps showing the uniformity of the measured beam shift in the horizontal and vertical directions. Color bar units are in $\mu \mathrm{rad}$. 
When examining the beam profile of the pink branch in the XRT and the FEH, a small beam steering on the order of $0.2 \mu \mathrm{rad}$ in the horizontal direction was observed. It originates from the refraction of the $\mathrm{x}$-ray beam from the front and back surfaces of the crystal that are not perfectly parallel as illustrated in Fig. 7. From profile measurement taken $200 \mathrm{~m}$ downstream of the diamond crystal using a scintillator screen, we observe a $\sim 50 \mu \mathrm{m}$ static shift of the horizontal beam profile, as shown in Fig. 8. The shift is much less pronounced in the vertical direction. The amount of horizontal steering $\Delta=\theta_{i}+\delta-\theta_{o}$ can be calculated following Snell's law, with which we deduced the angle $\delta$ between the two surfaces in the horizontal to be around $0.1^{\circ}$, consistent with the tolerance in the polishing process. However, as shown in Fig. 8, we did not observe significant beam intensity profile distortion in addition to the shift.

Because of the FEL shot-to-shot beam pointing fluctuations and the beam profile structure originating from the front end hard x-ray off-set mirrors, ${ }^{36}$ a detailed characterization of the wavefront distortion is challenging. We nevertheless examined the uniformity of the diamond crystal by mapping the amount of beam steering $200 \mathrm{~m}$ downstream from different part of the $2 \times 4 \mathrm{~mm}^{2}$ working area. The beam was slitted down to $50 \times 50 \mu \mathrm{m}^{2}$, and a $5 \times 5$ grid of locations was inspected as indicated in Fig. 9(a). The horizontal and vertical steerings are mapped out in Figs. 9(b) and 9(c). The distribution is rather uniform with a mean value around $0.25 \mu \mathrm{rad}$. It is especially the case in the center of the crystal where the working area indicated in Fig. 9 highlights the full incident beam foot print.

\section{CONCLUSION AND PROSPECT}

In conclusion, we have demonstrated the capability of splitting the LCLS x-ray FEL beam into a pink and a monochromatic branch to be used in two experimental hutches simultaneously. The high quality of the beamsplitting diamond crystal is reflected in the measured bandwidths and throughputs in both branches that closely match the theoretical values, as well as the minimally disturbed beam profile. The observed beam steering is attributed to the two surfaces of the thin diamond not being exactly parallel. This could be further minimized in the future by improving the polishing process.

To fully take advantage of the possibility of simultaneous experiments, one needs to better understand the additional operational constraints. In contrast to the one-experiment-ata-time scenario when the linac and undulators are optimized for a single experiment, the two simultaneous experiments need to agree on a common photon energy and x-ray pulse duration. Moreover, the common optics upstream from both instruments need to have settings that accommodate both experiments.

Newly developed and future FEL operation modes will further increase the flexibilities of the beam multiplexing scenarios by tailoring the spectral property of the beam. When the machine operates in the self-seeded mode with an enhanced SASE background, one could align the monochromator energy to the seeded line to obtain an increased flux in the monochromatic branch with some compromises of the flux in the pink branch. ${ }^{31}$ A more exciting development is the twocolor operation. ${ }^{37}$ One could envision the monochromatic branches picking up one bright spectral line while the FEL simultaneously delivers another tunable spectral line for the pink branch experiment, potentially creating an equal splitting scenario.

\section{ACKNOWLEDGMENTS}

B. Stephenson and L. Young are acknowledged for their support and interest in this work. Portions of this research were carried out at the Linac Coherent Light Source (LCLS) at SLAC National Accelerator Laboratory. LCLS is an Office of Science User Facility operated for the U.S. Department of Energy Office of Science by Stanford University. The presented work was also supported through a research grant from the Russian Ministry of Education and Science (Contract No. 16.552.11.7014). Effort in pre-characterization of the diamond crystals at the Advanced Photon Source was supported by the US Department of Energy, Office of Science, under Contract No. DE-AC02-06CH11357.

${ }^{1}$ P. Emma, R. Akre, J. Arthur, R. Bionta, C. Bostedt, J. Bozek, A. Brachmann, P. Bucksbaum, R. Coffee, F. J. Decker et al., Nature Photon. 4, 1-7 (2010).

${ }^{2}$ T. Ishikawa, H. Aoyagi, T. Asaka, Y. Asano, N. Azumi, T. Bizen, H. Ego, K. Fukami, T. Fukui, Y. Furukawa et al., Nature Photon. 6, 540 (2012).

${ }^{3}$ L. Young, E. P. Kanter, B. Krässig, Y. Li, A. M. March, S. T. Pratt, R. Santra, S. H. Southworth, N. Rohringer, L. F. DiMauro et al., Nature (London) 466, 56 (2010).

${ }^{4}$ H. N. Chapman, P. Fromme, A. Barty, T. A. White, R. A. Kirian, A. Aquila, M. S. Hunter, J. Schulz, D. P. DePonte, U. Weierstall et al., Nature (London) 470, 73 (2011).

${ }^{5}$ S. M. Vinko, O. Ciricosta, B. I. Cho, K. Engelhorn, H. K. Chung, C. R. D. Brown, T. Burian, J. Chalupský, R. W. Falcone, C. Graves et al., Nature (London) 482, 59 (2012).

${ }^{6}$ N. Rohringer, D. Ryan, R. A. London, M. Purvis, F. Albert, J. Dunn, J. D. Bozek, C. Bostedt, A. Graf, R. Hill et al., Nature (London) 481, 488 (2012).

${ }^{7}$ S. Boutet, L. Lomb, G. J. Williams, T. R. M. Barends, A. Aquila, R. B. Doak, U. Weierstall, D. P. DePonte, J. Steinbrener, R. L. Shoeman et al., Science 337, 362 (2012).

${ }^{8}$ S. O. Hruszkewycz, M. Sutton, P. H. Fuoss, B. Adams, S. Rosenkranz, K. F. Ludwig, W. Roseker, D. Fritz, M. Cammarata, D. Zhu et al., Phys. Rev. Lett. 109, 185502 (2012).

${ }^{9}$ N. D. Loh, C. Y. Hampton, A. V. Martin, D. Starodub, R. G. Sierra, A. Barty, A. Aquila, J. Schulz, L. Lomb, J. Steinbrener et al., Nature (London) 486, 513 (2012).

${ }^{10}$ T. E. Glover, D. M. Fritz, M. Cammarata, T. K. Allison, S. Coh, J. M. Feldkamp, H. Lemke, D. Zhu, Y. Feng, R. N. Coffee et al., Nature (London) 488, 603 (2012).

${ }^{11}$ S. Bernitt, G. V. Brown, J. K. Rudolph, R. Steinbrügge, A. Graf, M. Leutenegger, S. W. Epp, S. Eberle, K. Kubiček, V. Mäckel et al., Nature (London) 492, 225 (2012).

${ }^{12}$ J. Kern, R. Alonso-Mori, R. Tran, J. Hattne, R. J. Gildea, N. Echols, C. Glöckner, J. Hellmich, H. Laksmono, R. G. Sierra et al., Science 340, 491 (2013).

${ }^{13}$ M. Dell'Angela, T. Anniyev, M. Beye, R. Coffee, A. Föhlisch, J. Gladh, T. Katayama, S. Kaya, O. Krupin, J. LaRue et al., Science 339, 1302 (2013).

${ }^{14}$ J. N. Clark, L. Beitra, G. Xiong, A. Higginbotham, D. M. Fritz, H. T. Lemke, D. Zhu, M. Chollet, G. J. Williams, M. Messerschmidt et al., Science 341, 56 (2013).

${ }^{15}$ J. Als-Nielsen, A. K. Freund, G. Grübel, J. Linderholm, M. Nielsen, M. S. del Rio, and J. P. F. Sellschop, Nucl. Instrum. Meth. Phys. Res. B 94, 306 (1994).

${ }^{16}$ Y. Tsusaka, K. Yokoyama, S. Takeda, K. Takai, Y. Kagoshima, and J. Matsui, Nucl. Instrum. Meth. Phys. Res. A 467-468, 670 (2001).

${ }^{17}$ A. Robert, R. Curtis, D. Flath, A. Gray, M. Sikorski, S. Song, V. Srinivasan, and D. Stefanescu, J. Phys.: Conf. Ser. 425, 212009 (2013). 
${ }^{18}$ S. Lee, W. Roseker, C. Gutt, Z. Huang, Y. Ding, G. Grübel, and A. Robert, Opt. Exp. 20, 9790 (2012).

${ }^{19}$ S. Boutet and G. J. Williams, New J. Phys. 12, 035024 (2010).

${ }^{20}$ Y. Feng, D. Zhu, A. Robert, H. T. Lemke, M. Chollet, D. M. Fritz, J. B. Hastings, J. M. Feldkamp, M. Cammarata, S. Moeller et al., Proc. SPIE 8504, 85040V (2012).

${ }^{21}$ H. Yamaoka, K. Ohtomo, and T. Ishikawa, Rev. Sci. Instrum. 66, 2116 (1995).

${ }^{22}$ G. Grübel, D. Abernathy, G. Vignaud, M. Sanchez del Rio, and A. Freund, Rev. Sci. Instrum. 67, 3349 (1996).

${ }^{23}$ P. B. Fernandez, T. Graber, W. K. Lee, and D. M. Mills, Nucl. Instrum. Meth. Phys. Res. B 400, 476 (1997).

${ }^{24}$ R. C. Burns, A. I. Chumakov, S. H. Connell, D. Dube, H. P. Godfried, J. O. Hansen, J. Härtwig, J. Hoszowska, F. Masiello, L. Mkhonza et al., J. Phys.: Condens. Matter. 21, 364224 (2009).

${ }^{25}$ Y. V. Shvyd'ko, S. Stoupin, A. Cunsolo, A. H. Said, and X. Huang, Nature Phys. 6, 196 (2010).

${ }^{26}$ Y. Shvyd'ko, S. Stoupin, V. Blank, and S. Terentyev, Nature Photon. 5, 539 (2011).

${ }^{27}$ S. N. Polyakov, V. N. Denisov, N. V. Kuzmin, M. S. Kuznetsov, S. Y. Martyushov, S. A. Nosukhin, S. A. Terentiev, and V. D. Blank, Diamond Relat. Mater. 20, 726 (2011).

${ }^{28}$ H. Sumiya and K. Tamasaku, Jap. J. Appl. Phys. 51, 090102 (2012).

${ }^{29}$ S. Stoupin, V. D. Blank, S. A. Terentyev, S. N. Polyakov, V. N. Denisov, M. S. Kuznetsov, Y. V. Shvyd'ko, D. Shu, P. Emma, J. Maj et al., Diamond Relat. Mater. 33, 1 (2013).
${ }^{30}$ J. Horbach, M. Degenhardt, U. Hahn, J. Heuer, H. B. Peters, H. SchulteSchrepping, A. Donat, and H. Lüdecke, Nucl. Instrum. Meth. Phys. Res. A 649, 136 (2011).

${ }^{31}$ J. Amann, W. Berg, V. Blank, F. J. Decker, and Y. Ding, Nature Photon. 6, 693 (2012).

${ }^{32}$ Y. Feng, R. Alonso-Mori, V. Blank, S. Boutet, M. Chollet, T. B. van Driel, D. M. Fritz, J. M. Glownia, J. B. Hastings, H. Lemke et al., Proc. SPIE 8778, 87780B (2013).

${ }^{33}$ S. Stoupin, S. A. Terentyev, V. D. Blank, Yu. V. Shvyd'ko, K. Goetze, L. Assoufid, S. N. Polyakov, M. S. Kuznetsov, N. V. Kornilov, J. Katsoudas, R. Alonso-Mori, M. Chollet, Y. Feng, J. M. Glownia, H. Lemke, A. Robert, S. Song, M. Sikorski, and D. Zhu, "All-diamond optical assemblies for a beam-multiplexing X-ray monochromator at the Linac Coherent Light Source," preprint arXiv:1401.5879v1 (2014).

${ }^{34}$ D. Zhu, M. Cammarata, J. M. Feldkamp, D. M. Fritz, J. B. Hastings, S. Lee, H. T. Lemke, A. Robert, J. L. Turner, and Y. Feng, Appl. Phys. Lett. 101, 034103 (2012).

${ }^{35}$ Y. Feng, J. M. Feldkamp, D. M. Fritz, M. Cammarata, R. Aymeric, C. Caronna, H. T. Lemke, D. Zhu, S. Lee, S. Boutet et al., Proc. SPIE 8140 , 81400Q (2011).

${ }^{36}$ S. Rutishauser, L. Samoylova, J. Krzywinski, O. Bunk, J. Grünert, H. Sinn, M. Cammarata, D. M. Fritz, and C. David, Nature Commun. 3, 947 (2012).

${ }^{37}$ A. A. Lutman, R. Coffee, Y. Ding, Z. Huang, J. Krzywinski, T. Maxwell, M. Messerschmidt, and H. D. Nuhn, Phys. Rev. Lett. 110, 134801 (2013). 